\title{
Use of Statecharts in the Modelling of Dynamic Behaviour in the ATLAS DAQ Prototype -1
}

\author{
P. Croll ${ }^{1}$, P.-Y. Duval ${ }^{2}$, R. Jones ${ }^{3}$, S. Kolos ${ }^{4}$, R.F. Sari ${ }^{1}$ and S. Wheeler ${ }^{1}$ \\ ${ }^{1}$ University of Sheffield, Western Bank, Sheffield, S10 2TN, UK \\ ${ }^{2}$ Centre de Physique des Particules de Marseille, 163 avenue de Luminy, case 907, 13288 Marseille cedex 09, France \\ ${ }^{3}$ CERN, ECP Division, CH-1211 Geneva 23, Switzerland \\ ${ }^{4}$ Petersburg Nuclear Physics Institute, Gatchina, Leningrad district, 188350 Russia (on leave at CERN)
}

\begin{abstract}
Many applications within the ATLAS DAQ prototype-1 system have complicated dynamic behaviour which can be successfully modelled in terms of states and transitions between states. Previously, state diagrams implemented as finite-state machines have been used. Although effective, they become ungainly as system size increases. Harel statecharts address this problem by implementing additional features such as hierarchy and concurrency.

The CHSM object-oriented language system is freeware which implements Harel statecharts as concurrent, hierarchical, finite-state machines (CHSMs). An evaluation of this language system by the ATLAS DAQ group has shown it to be suitable for describing the dynamic behaviour of typical DAQ applications. The language is currently being used to model the dynamic behaviour of the prototype-1 run-control system. The design is specified by means of a CHSM description file, and $\mathrm{C}++$ code is obtained by running the CHSM compiler on the file. In parallel with the modelling work, a code generator has been developed which translates statecharts, drawn using the StP CASE tool, into the CHSM language. $\mathrm{C}++$ code, describing the dynamic behaviour of the run-control system, has been successfully generated directly from StP statecharts using the CHSM generator and compiler. The validity of the design was tested using the simulation features of the Statemate CASE tool.
\end{abstract}

\section{INTRODUCTION}

ATLAS is a particle physics experiment under construction for the Large Hadron Collider (LHC) at CERN. The DAQ system foreseen for this experiment will have to be able to reduce the unprecedented data rates $(\sim 10$ GByte/s) to a level ( $100 \mathrm{Mbyte} / \mathrm{s})$ at which interesting events can be written to mass storage. This must be done without losing any new or previously unpredicted physics. In order to meet the challenge, the ATLAS DAQ/EF (Data Acquisition / Event Filter) "prototype-1" project aims to produce a fully-functional prototype suitable for evaluating candidate architectures and technologies for the final $\mathrm{DAQ} / \mathrm{EF}$ system of the experiment. The prototype consists of a complete "vertical slice" of the ATLAS DAQ/EF architecture. It includes all the hardware and software elements of the data-flow chain, and the control and monitoring required in an on-line system.

Within the prototype project, the back-end DAQ [1] encompasses the software needed to configure, control and monitor the DAQ, but excludes the management, processing and transportation of physics data.

The run-control system is one of the software components of the back-end DAQ. It is responsible for controlling the data-taking activities of acquisition components throughout the DAQ system. It can send commands to DAQ components, query or receive status information from them and provides operators with a means to act upon the DAQ system.

The ATLAS collaboration has defined requirements for a software process and a supporting environment to develop and modify all ATLAS software [2]. CASE (Computer Aided Software Engineering) tools using suitable objectoriented modelling methods have been identified as an appropriate technology for supporting many aspects of this process.

This paper begins with a summary of the process, presented in more detail elsewhere $[3,6]$, by which suitable methods and CASE tools for the design of the run-control system and other DAQ software components were chosen. An overview of the chosen CASE tools is given. This is followed by a more detailed description of the method by which these tools have been used to achieve and subsequently verify the high-level design of the ATLAS DAQ prototype run-control system.

\section{ChOICE OF METHOD AND CASE TOOLS}

The ATLAS experiment will demand software production on a scale far beyond that previously addressed in High Energy Physics (HEP). Due to its size, complexity and foreseen life-time, it is crucial that software is produced and maintained to the highest possible engineering standards. A considerable shift from the methods traditionally used to produce software in HEP is required. Fortunately, advances in computer science and technology have provided software developers with powerful new tools to face this challenge. Object-oriented $(\mathrm{OO})$ design methods used in conjunction with CASE tools allow the production of robust, re-usable software, with sufficient flexibility to meet the changing requirements of an HEP experiment. This section summarises the process by which a suitable $O O$ method and supporting CASE tools were chosen to design the run-control and other software systems in the DAQ prototype project.

Many applications within the ATLAS DAQ system, including the run-control system, exhibit complicated dynamic behaviour. The system can be successfully 
modelled in terms of states and transitions between states. In the past, a number of different methods for modelling such complex behaviour have been reviewed and/or used. These methods are Petri nets, Z-Specification and State Diagrams.

Petri nets offer good concurrent modelling facilities with a graphical representation that has a formal mathematical equivalence. The basic Petri net suffers by requiring low-level detail to capture a complete system model. Analysis of any non-trivial system can suffer from state explosion, i.e., the search space is an exponential of the system states. Petri net based CASE tools, e.g. Design/CPN, handle this through substitution transitions (hierarchy) and colours (data typing). Experience has shown that these tools need considerable skill from a software engineer plus constraints on the design to make analysis tractable [3]. Petri net tools also lacked dependable commercial support.

$Z$ is a set-based formal method that permits unambiguous mathematical specification but has no support for concurrency and like many formal mathematical methods, does not support graphical simulation.

State diagrams allow the dynamics of a system to be modelled in terms of system states and transitions between states. However, modelling large systems becomes ungainly due to a lack of abstraction. Harel statecharts [4] address this through introducing state hierarchy and concurrency. Using the OMT modelling method [5] allows the static structure to be represented via an object model while Harel statecharts provide modelling of dynamic behaviour. The aim of using CASE tools for diagrammatical representations and modelling is to allow software developers to concentrate on the design aspects rather than implementation.

When choosing a CASE tool for the design of the runcontrol software, the following points also had to be taken into consideration:

- It should be possible to generate code automatically from the design diagrams to ensure the maintainability of the software over several versions.

- The run-control system has to run in a heterogeneous environment. The software developed with the tool will have to compile and run correctly on different operating systems, presently Solaris, LynxOS, HPUX and WindowsNT. Hence code developed with the tool should be portable.

- It must be possible to customise the code generation so that different languages can be produced for all elements of the model (i.e. not just from the object model), and in order that differences in compilers on different machines can be taken into account

- It must be possible to integrate generated code with third-party software.

- The tool should be lightweight, not requiring excessive resources to run.

- The tool should be easy to learn and to use.
After evaluations of different CASE tools [6], it was decided that the StP/OMT (Software through Pictures) CASE tool [7] was the best overall product satisfying the above conditions.

Although code generators can be written and integrated with $\mathrm{StP}$, allowing $\mathrm{C}++$ code to be generated directly from the dynamic model, an intermediate step, using the CHSM object-oriented language system [8] has been employed which greatly simplifies the effort. The CHSM language system is summarised in the following section.

\section{OVERVIEW OF THE CASE TOOLS}

Since StP and its code-generation capabilities are described in detail elsewhere [9], only CHSM will be described here.

CHSM is a theoretically-rigorous, object-oriented language system, built on $\mathrm{C}++$, which implements Harel statecharts as Concurrent, Hierarchical, Finite-State machines. The language system supports the following statechart concepts:

- Hierarchy: child states can be "nested" in parent states, allowing the parent state to be treated as a "black-box".

- Clusters: logical-exclusive-or state groups eliminate the need for replicated transitions.

- Sets: logical-and state groups eliminate the exponential increase in the number of states when new states are added.

- Concurrency: sets allow transitions caused by the same event to occur simultaneously in different parts of the statechart.

- History: entering a cluster enters the child-state that was last active (as opposed to the default child-state)

- Guard conditions: transitions are only made if a predefined condition is true.

- Actions: actions can be executed when transitions are made and on entering or exiting a state.

- Broadcasting: events can be broadcast when a transition is made.

- Implicit broadcasting: events are broadcast every time states are entered or exited possibly triggering transitions in other parts of the statechart.

The above features can be described in a CHSM description file. This is an ordinary text file consisting of three sections. The first section contains declarations required by any $\mathrm{C}++$ code embedded in the rest of the file. The second is the CHSM description itself and the third contains optional user code. Guard conditions, transition and state enter and exit actions are specified in the middle section of the description file with the necessary $\mathrm{C}++$ code.

The description file is converted to $\mathrm{C}++$ by the CHSM compiler. The resulting code is compiled and linked with the CHSM run-time library, the source for which is freely available. In the CHSM run-time library, states, clusters and sets are implemented as $\mathrm{C}++$ classes. These classes have 
predefined data-members and member-functions. The user can derive classes from these classes, using $\mathrm{C}++$ inheritance to add data-members and member-functions. Furthermore, existing methods, such as state enter and exit functions, can be overloaded in order to augment their behaviour. This is a powerful feature allowing $\mathrm{C}++$ classes used in the DAQ system to inherit their dynamic behaviour from CHSM.

There are several independent groups working on the DAQ sub-systems. They will need to customise the controller responsible for their part of the DAQ to perform the operations specific for their particular component. The actions which will need to be performed cannot be defined in advance. A generic statechart is defined using StP and CHSM which the developers use as a "template" framework into which they can add their own specific operations.

Evaluations in the ATLAS DAQ group have proved CHSM to be a very robust and flexible tool. It is a hybrid language, where $\mathrm{C}++$ has been extended with additional constructs and, as such, it has proved to be quick and easy to learn. Furthermore, since it is based on $\mathrm{C}++$, the resultant code is easily incorporated with other programs and modules of the DAQ system. A CHSM code generator has been integrated with the StP tool to allow CHSM description files to be generated automatically from statecharts in the tool. The code generated by the CHSM compiler, as well as the source for the run-time library, compiles and runs correctly on all the platforms foreseen in the DAQ system. It has also been shown that programs can be written which successfully combine the CHSM code with code from other commercial tools to be used in the DAQ system (e.g. Corba/ILU, X-Window System, Rogue Wave Tools.h++ C++ class library etc.)

\section{ARCHTTECTURE OF THE RUN CONTROL}

Due to the size and complexity of the ATLAS experiment, the run-control system cannot be implemented as a single program. It is foreseen that the system will consist of many programs executing on several distributed computers connected through a network, as is already the case with the Aleph experiment at LEP [10]. Such a distributed system reflects the structure of the DAQ itself and will be implemented as a hierarchy of entities called controllers, each with responsibility for a well-defined component of the DAQ system. The controller's state is the simplified external view of the current working condition of the component under its responsibility.

Each controller can receive commands from the outside world. Commands cause a controller to execute actions which potentially change the state of the controlled component. The state of the component is published by the controller to make it "visible" to the outside world. A controller can also react to local events occurring in the component under its responsibility. Typically its reaction will be to execute some actions and potentially change its visible state.

The controllers are organised into a hierarchical tree structure that reflects the general organisation of the DAQ system itself. The hierarchy is defined in a configuration database which is described in detail elsewhere [11]. Each controller in the tree can have one parent (or superior) controller and any number of child (subordinate) controllers. At the top of the tree is a single controller which represents the overall state of the entire system.

The controllers in the hierarchical tree transmit messages between each other over a local-area network (using the OMG Corba standard implemented by ILU [12]) in order to exchange commands and status information. In general, commands starting from the human operator are sent to the overall controller, which forwards them to the sub-system controllers, who in turn forward them to component controllers and so on. In this respect commands flow down from the root of the tree towards the leaves. Replies, indicating the successful completion or otherwise of commands and state information, are sent back up the tree so that the human operator is made aware of any change in the state of the system, or of any errors which have occurred. Any node in the control tree can perform actions on the commands or results of commands it receives.

\section{HIGH-LEVEL DESIGN OF THE RUN-CONTROL SYSTEM}

The high-level design of the dynamic behaviour of a single run controller was accomplished using the StP CASE tool. Two statecharts were drawn reflecting different aspects of a run controller's behaviour. It is envisaged that every controller in the hierarchy will be modelled by the same two statecharts. The "Manager CHSM" handles the way in which a controller interacts with the other controllers within the run control hierarchy. It also forwards commands it receives from the operator or parent controller to the second "generic DAQ controller CHSM". This CHSM models the state of the controlled component and will try to execute the forwarded command, possibly causing a change of state. Once command execution is complete the generic CHSM reports the success or failure to the manager CHSM which then takes appropriate action.

In more detail, the generic DAQ controller CHSM models the sequence of events necessary to take the controlled apparatus from an idle state, where no data are being taken, to an active, running state where data are being collected from the apparatus, and back again. The CHSM, extracted from StP, is shown in Figure 1. State changes are initiated by commands corresponding to events on this statechart. These are sent by a human operator and then propagated through the run control system. It is foreseen that developers of the various controllers will be able to customise the behaviour of their particular controller by adding code to implement the required actions within the generalised template provided by this CHSM.

Referring to Figure 1, the central Alive super-state is composed of two concurrent states: DAQActivity, which reflects the current status of data-taking activities and DAQFault, which reflects whether or not an error has occurred in the component being controlled. The human operator issues commands which correspond to events in the DAQActivity state. When transitions, caused by these 
events, occur, actions are performed which carry out the necessary operations to take the controlled component from one state to the next. In the tool, the transitions are labelled using standard OMT notation in the following manner: event_name[guard_condition]/action. The CHSM language system is used to specify the guard conditions and actions. On the diagram only event_name is shown for clarity.

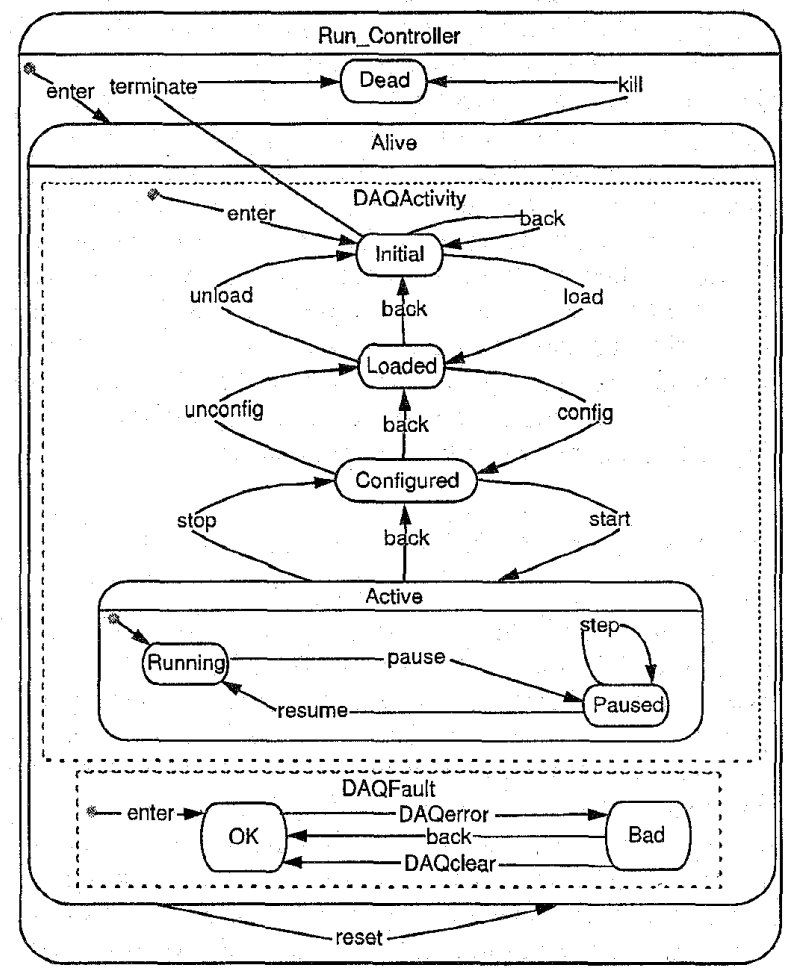

Figure 1: Simplified generic run controller CHSM extracted from the StP/OMT CASE tool.

If the action fails for some reason (i.e. the component being controlled does not respond correctly to the action), this can be signalled to the parent controller by issuing the $D A Q e r r o r$ event which takes the concurrent DAQFault state to $B a d$. Recovery mechanisms have been envisaged for three different levels of error. Firstly, if an error occurs when making a transition between two states, a mechanism is foreseen to take the CHSM back to the last error-free state undoing any actions which were made during the transition which caused the error. Secondly, if the error is more serious and cannot be cleared by the above mechanism, the whole CHSM can be reset, during which, all allocated resources are reset and freed and the CHSM is put back to its initial state. Finally, if a fatal, nonrecoverable error occurs somewhere in the overall run control system, the whole system can be shut down as cleanly as possible. Since system integrity cannot be guaranteed in such a situation, individual controllers cannot rely on any communication or external interaction during the shutdown.

The Manager CHSM models the interaction of the controller with the other controllers in the run-control hierarchy. The Manager CHSM, after being ported to Statemate, is shown in Figure 2. The port was done in order to make use of Statemate's powerful test facilities (see section VI). For communication between controllers, dedicated $\mathrm{LU}$ is used for sending commands down the tree and the back-end DAQ Information Service [13] for returning replies up the tree and for making DAQActivity states visible to outside parties.

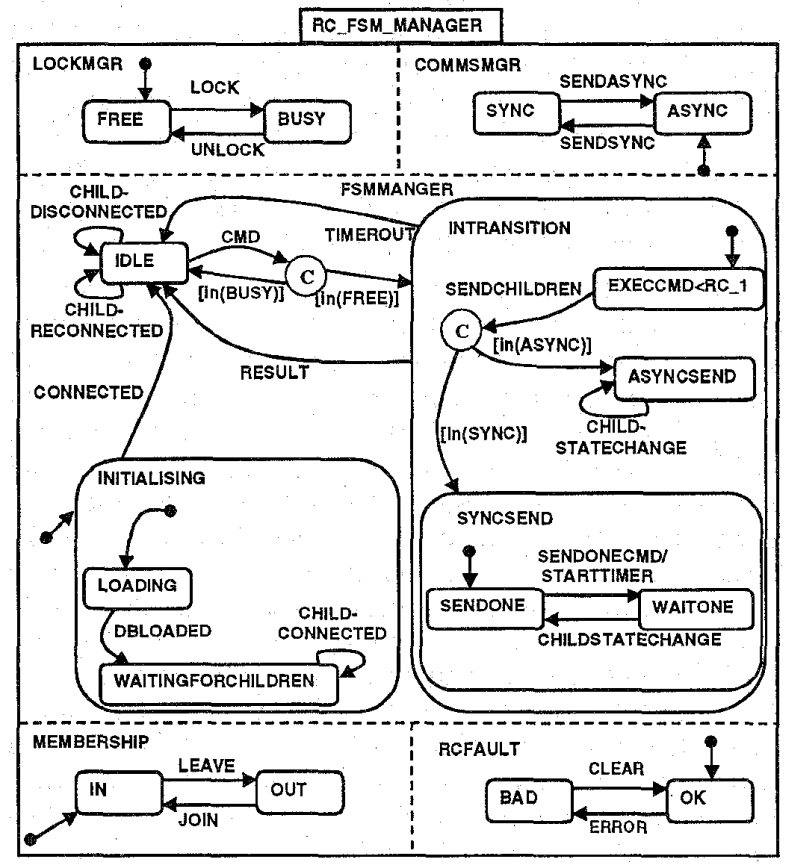

Figure 2: Simplified run controller manager CHSM extracted from the Statemate CASE tool.

The main purpose of the Manager CHSM is to handle the initial configuration of a controller when booting, and to marshal the generic CHSM through transitions when DAQ control commands are received from the operator or from a parent controller. This includes forwarding the command to any children a controller may have-either in synchronous or asynchronous mode depending on the nature of the action being carried out. During the initialisation and transition phases, the CHSM is locked so that no new commands can be received.

A concurrent state called RCFault is used to reflect the status of the run control hierarchy itself. If a controller has a problem with any of its children (e.g. a child is dead, not responding,etc.) the RCFault state is set to $\mathrm{Bad}$.

The Membership concurrent state indicates whether the controller is part of the run control hierarchy of controllers. When In the controller will be controlled by its parent controller; it will be ignored when it is Out. This feature allows a controller with a specific problem needing attention to be isolated without affecting the rest of the system.

A similar method has been used to design and implement the DAQ supervisor. This is another component of the run-control system, responsible for the creation and 
supervision of all the software elements in the run-control hierarchy.

\section{TESTING THE HIGH-LEVEL DESIGN}

Although the approach taken has allowed a high-level design of the run control to be created covering all aspects of the OMT model, there was no simple way in which the dynamic model could be simulated to test its validity before implementation. To overcome this two different methods of testing were investigated. Firstly, porting the statecharts to the Statemate CASE tool and then using its powerful simulation facilities for animation. Secondly, the CHSM code generator integrated with StP was used to generate a prototype and then the CHSM run-time library debugger was used to check the behaviour.

\section{A. Usage of the Statemate CASE Tool}

The Statemate "Magnum" CASE tool [14] has been used as a simulation tool in testing the high-level design in the ATLAS project. Statemate contains a set of graphical tools for the specification, analysis, design and documentation of large and complex reactive systems. Activity charts provide a functional description, Module charts structural description and Statecharts the behaviour.

Statemate aims to be an industrial-strength CASE tool and includes robust graphical chart editors and a userdefined control panel, plus simulation and dynamic test facilities. An automatic code generator produces $\mathrm{C}$ and ADA directly from the graphical models. Analysis of the statechart models allows issues of reachability, deadlock and transitions usage to be addressed.

Statemate uses syntax similar to the CHSM language system to label a transition in a statechart, i.e. "event[condition]/action". Some minor differences in syntax between the CHSM and Statemate statecharts were found during the prototype implementation.

Although the Activity Chart and Module Chart are available in Statemate, only the Statechart view was used to match the existing designs in StP and CHSM. The statechart behavioural view describes the system's behaviour over time, including the dynamics of activities, their control and timing behaviour, both the states and modes of the system and the conditions and events that cause mode and state changes. The behavioural model provides answers to questions about causality, concurrency and synchronisation, which are paramount features for the run-control system.

Statemate incorporates a broadcast communication mechanism, and time-out and delay operators for specifying synchronisation and timing information. Each element in the statechart has an entry in the data dictionary, which can be used to input additional specific information. Statemate allows large charts to be split into separate hierarchical ones as seen by the two communicating statecharts, of Generic Run Controller and RC_FSM_Manager, see Figures 1 and 2. As with programming abstractions, this facility improves readability through structure hiding and promotes chart reuse.
The Statemate data-dictionary editor has been used to configure the binding of the statechart objects and to create an array of objects to show which instantiations need to be controlled. It can also be used to configure the data structure, data type and usage of an object.

\section{B. Simulation with Statemate}

The dynamic behaviour can be simulated, allowing visual observation of the changing transitions and states through the use of different colours. Before a simulation can be run the scope of the simulation profile will check the correctness of the statecharts to detect inconsistencies, while a completeness check detects redundancy and any incompleteness in a model. Hence, possible violation in syntax and semantics, both in a single chart and in communicating charts, can be detected.

\section{Lessons Learnt from Statemate}

The approach taken enabled binding of different object names by using the instantiation features of Statemate for simulating different statecharts. However, the implementation of the system in a distributed environment and with real hardware devices has still to be validated. Not all the features of the hierarchy of control have been simulated and analysed. More experiments are needed to test the use of the hierarchy of statecharts in the run-control system, i.e. would it be possible to model the interaction between controllers in the hierarchy and not just between the statecharts.

Some benefits of Statemate Magnum in enhancing the overall software engineering cycle have been observed during this project. For example, it simplifies modelling of a complex system, it eliminates ambiguities common in textual specification, it validates system behaviour, and it detects and eliminates specification errors before implementation.

The most helpful feature of Statemate, in this study; is its capability to simplify the understanding of operation with clear animation of graphical models. CASE tools such as Statemate can be used to show what would happen if multiple interacting events take place, which is sometimes hard to conceptualise.

\section{Testing with CHSM}

The CHSM code generator integrated with StP was used to create $\mathrm{C}++$ code corresponding to the high-level design. The code was compiled and linked with the CHSM run-time library in order to get a working test program. CHSM provides tracing facilities that print output to the screen including currently active states and information on the event handling. Using these debug features, it was found, for instance, that the concurrent state in the Manager CHSM, responsible for locking the CHSM when handling a transition, was not synchronised properly. Unfortunately, in order to carry out more extensive tests, it has been necessary to add a significant amount of hand-written code to implement the controller hierarchy. 


\section{CONCLUSIONS}

A combination of CASE tools that support statecharts to create and test the high-level run control design were utilised. Harel statecharts were found to be an appropriate method for modelling the dynamic behaviour of the run control. StP provided the necessary formalism and its code generation facilities, combined with the CHSM language system, gave sufficient flexibility to allow third-party software to be successfully integrated. This enabled the generated software to run in a heterogeneous environment and allowed each controller in the system to be customised. The debugging facilities of the CHSM language system, combined with the simulation facilities of Statemate, allowed testing of the high-level design before embarking on the detailed implementation. Now that the high-level design is complete, a prototype run controller has been created and integrated with third-party software to implement communication between controllers and is currently undergoing thorough testing.

For future work the possibility of automatically porting the design from StP to Statemate is being investigated. For the current project, the porting was done by hand. One problem encountered with Statemate is how to simulate the distributed run controller hierarchy. Although validation for a single run controller model was achieved, creating a simulation of the hierarchy proved more difficult, although this should be possible. This would involve multiple copies of the run controller statecharts, representing different processes running on different machines, interacting in a hierarchy.

Rhapsody, a successor to Statemate, is a new CASE tool launched in May 1997 by i-Logix as an integrated set of diagrammatic languages for object modelling [15]. It is still built around statecharts, but holds promise to overcome some of the difficulties that have been have encountered. The plan is to investigate the use of Rhapsody in forthcoming ATLAS Trigger/DAQ activities.

\section{ACKNOWLEDGEMENTS}

We would like to thank Paul J. Lucas for answering many questions about the CHSM language-system and his considerable help in porting the system to WindowsNT. We would also like to thank Kivanc Nurdan and Louis Tremblet for their input on the design of the run controller.

\section{REFERENCES}

[1] ATLAS DAQ Back-end software - User Requirements Document, 30 May 1996. http://atddoc.cern.ch/Atlas/DaqSoft/document/draft_1.h tml.

[2] ATLAS Software Development Environment - User Requirements Document, 26 April 1996. ATLAS Internal Note SOFT-NO-034, also available from http://atlasinfo.cern.ch/Atlas/documentation/notes/SOF TWARE/note34/asde.html
[3] P.R. Croll, I.E. Jelly and I. Gorton, "Software Engineering Techniques and Tools for High Performance Parallel Systems", IEEE Aizu International Symposium on Parallel Algorithm/Architecture Synthesis (pAs'95), Japan, 1995, pp. 85-91.

[4] D. Harel, "Statecharts: A Visual Formalism for Complex Systems", Sci. Computer Prog., pp. 231-274, July 1987.

[5] James Rambaugh, Michael Blaha, William Premerlani, Frederick Eddy, William Lorensen, Object Oriented Modeling and Design, Prentice Hall, 1991.

[6] C. Bee et al., "Applications of an OO methodology and CASE to a DAQ system", Proceedings of the International Conference on Computing in High Energy Physics 95, World Scientific Publishing, 1996, pp. 678686.

[7] Information on StP/OMT is available on WWW at: http://www.ide.com/Products/StP/StP_OMT.html.

[8] P. J. Lucas, F. Riccardi, "CHSM: A Language System Extending $\mathrm{C}++$ for Implementing Reactive Systems", http://www.best.com/ pjl/software.html.

[9] A. Patel et al., "Use of Object oriented CASE tools for Automating the Development of DAQ Software", to be published in Proceedings of the $10^{\text {th }}$ IEEE-NPSS Real Time Conference, Beaune, 1997.

[10]A.Aimar et al., "Representing system behaviour with dynamic models and executing them: The new Aleph finite state machine", Proceedings of the International Conference on Computing in High Energy Physics 95, World Scientific Publishing, 1996, pp. 625-631.

[11]I. Soloviev et al,; "The Persistent In-memory Object Manager", to be published in Proceedings of the $10^{\text {th }}$ IEEE-NPSS Real Time Conference, Beaune, 1997.

[12]A. Amorim et al., "Use of Corba in the ATLAS prototype DAQ", IEEE Trans. On Nucl. Science, vol. 45, No. 4, August 1998.

[13] M. Caprini et al., "Information service for Atlas DAQ Prototype -1", ATLAS DAQ Prototype-1 Technical Note 31.

http://atddoc.cern.ch/Atlas/Notes/031/Note031-1.htmI

[14]D. Harel, "STATEMATE: A Working Environment for Development of Complex Reactive System", IEEE Transactions on Software Engineering, pp. 403-414, April 1997.

[15]D. Harel and E. Gery, "Executable Object Modeling with Statecharts", IEEE Computer Magazine, pp. 3142, July 1997. 